\title{
Frequency Regulation with the Lifespan of EVs Batteries
}

\author{
Yufeng Guo, Yi Jiang and, Jilai Yu \\ School of Electrical Engineering and automation \\ Harbin Institution of Technology \\ P.O.box 353 (Harbin, China) \\ Phone/Fax number:+0451 86413641, e-mail: mailto:guoyufenghit@163.com, mailto:1943178001@qq.com
}

\begin{abstract}
In this paper, the model of EVs batteries with lifespan factors is established. As one part of power grid, it is proved to be effective to the primary regulation. According to a power law equation in which the capacity loss of batteries follows the current, temperature and DOC/DOD with time,the model is established. In this model, current and DOC/DOD are the main factors.By using the data from the simulation of power grid, the capacity loss of different strategies can be analysed. When charging/discharging integrated, in one cycle, the capacity loss of EVs batteries can be obtained at room temperature.
\end{abstract}

\section{Key words}

Electric vehicle, Frequency regulation, Estimation method, Lifespan of batteries, Capacity loss

\section{Introduction}

Electric vehicles (EVs) can provide ancillary services, such as regulation services, to the utility grid depending on the control signal ${ }^{[1]}$. Comparing to the traditional frequency modulation reserve, EVs can response to the signal quickly and accurately. What's more, with the popularity of the charging stations, the scale of EVs is bigger. The capacity of EVs batteries can be sufficient for grid regulation $^{[2]}$. However, there is no doubt that quick response and frequent adjustment are harmful to the lifespan of EVs batteries.

In this paper, the regulation strategies with EVs, considering the lifetime of batteries are designed. In Section II, we analyse varieties of EVs batteries and the methods of estimating batteries lifespan. The factors are used to build the model of batteries in order to prolong the lifetime. And the capacity of batteries can be estimated. In Section III, on account of the actual system data, different strategies with EVs regulation are analysed how to influence the health of batteries. Finally, Section VI draws the conclusion.

\section{Batteries and Estimation methods}

\section{A. Batteries}

Common EVs batteries can be divided into five branches, lead-acid battery, nickel-metal hydride battery, lithium battery, fuel cell battery and super-capacitor, with different features. The technology of lead-acid battery is most mature above all. Comparing with its low cost, low energy density and high self-discharge rate limit its growth. Fuel cell battery has high specific energy and high energy conversion. However, transportation difficulties must be solved. The market of lithium battery holds the promising future on account of its high specific energy and wide material sources.

\section{B. Estimation Methods}

EVs lifespan includes two parts, cycle life and storage life. Nonetheless, the feature of frequent charging / discharging decides that the cycle life becomes the central branch of research. Experience-based method and performance-based method are adopted to estimate the cycle life of EVs batteries.

Experience-based method includes three main methods, cycle index method, weighted ampere-hour method and event-oriented method Cycle index method depends on times of cycle. Also, in different situations, assessment index will change. Weighted ampere-hour method shows that in the lifespan of the battery, charging and discharging ampere hour is constant. And the weight number will change with environmental situations. In event-oriented method, different issues reflect different influences on the lifespan of battery. When it occurs, battery lifetime will decrease ${ }^{[3]}$. It is based on experiments.

Performance-based method also contains three main parts, mechanism-based prediction, characteristics-based prediction and data-based prediction. By building electrochemistry model and single-particle model, mechanism-based prediction tries to find the influence of external factor on the lifetime of battery. In the process of aging battery, the capacity and resistance will change a lot. So characteristics-based prediction can measure SOC and impedance spectroscopy to estimate the lifetime of 
battery. Data-based prediction uses amount of experimental data to describe the character of batteries lifespan.

\section{Power Law Equation}

Capacity fade reflects the lifespan of batteries directly. There have been substantial efforts focusing on the development of models to predict capacity fade in lithium ion batteries. In this paper, we adopt to the prediction model from [4]. This model is based on the actual experimental data, considering four factors, cycle-index, temperature, depth of discharge and current. However, the factor we most concerned is current, which is proportional with power, for the voltage is constant(3.6V). Also, from the following simulation, the C-rates of batteries is low, so the formula we used is as follow.

$$
Q_{\text {Loss }}=B \times \exp \left(\frac{E_{a}}{R T}\right)(A h)^{z}
$$

$Q_{\text {Loss }}$ is the percentage of the batteries capacity loss, $B$ is the pre-exponential factor, $E_{a}$ is the activation energy, $R$ is the gas constant, $T$ is the absolute temperature, $A_{h}$ is the Ah-throughout ( $\mathrm{Ah}=$ cycle time) and $z$ is the power law factor $^{[4]}$.

The original model only considers the process of discharge. However, in the power grid, the EVs is bidirected transfer. In this paper, the process of charge is thought to be equivalent to the discharge, using the same model.

In addition, depending on the actual system, we consider 24 hours as one cycle time. However, the capacity of batteries changes moment by moment. We calculate 24 hours capacity loss with given depth of discharge first. Then, according to the time scale of the simulation, the capacity loss can be obtained in one period time, $\mathrm{t}$ (15 seconds). Finally, we can sum the results together to get the capacity in one cycle.

$$
\begin{aligned}
Q_{\text {Loss_periodtime_ } i}=B \times \exp \left(\frac{E_{a}}{R T}\right)\left(A h_{\text {period_time_i }_{-}}\right)^{z} \\
A h_{\text {period_time_ } i}=D O D \times 1 \times \frac{t}{86400} \text { or } \\
A h_{\text {period_time_ } i}=D O C \times 1 \times \frac{t}{86400} \\
Q_{\text {Loss }}=\sum_{i=1}^{n} Q_{\text {Loss_periodtime }_{-} i}
\end{aligned}
$$

In this paper, we take $25^{\circ} \mathrm{C}(298 \mathrm{~K})$ as the standard temperature. The experimental data provides us two points for the parameter $B$.So the linear relevant fitting result is as follow.

$$
B_{i}=-\frac{22000}{3} C_{-} \text {rate }_{i}+\frac{101900}{3}
$$

In addition, according to the fitting result, $E_{a}$ is equal to -31500 . The parameter, $z$ changes from 0.551 to 0.554 . Thus, $\mathrm{z}$ is equal to 0.553 .

\section{Strategies and Simulation}

In actual power system, change of frequency is very small. The character of battery lifetime can be linearized the curve locally at the rated frequency. The loads change moment by moment, therefore, frequency of power grid is in ever-changing situation continuously. In order to avoid slight fluctuation, dead zone is set. In addition, the limit of power can avoid the battery in unhealthy situations, such as over-charging, over-discharging, etc. These adverse conditions may destroy the physical structure and impact lifespan irreversible ${ }^{[4]}$. Considering these factors, the corresponding control block diagram can be built as figure 1 .

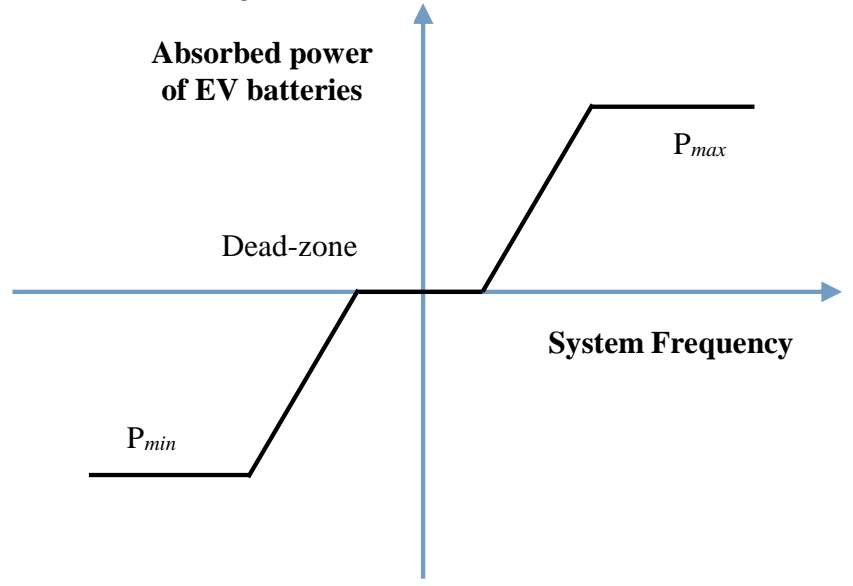

Fig.1. Ideal battery power-frequency characteristic curve

In order to reflect complexity of actual power system, single-region power grid model includes three generators with different generator frequency coefficients, zero bands and power distributions, all control primary frequency. What's more, two generators take part in secondary frequency control ${ }^{[5]}$. This part adopts PI program. The control block diagram is as figure 2. Step signal is used to make certain that the model is correct. Then the data from Huabei power grid is used to simulate. The frequency characteristic curve is as figure 3 .

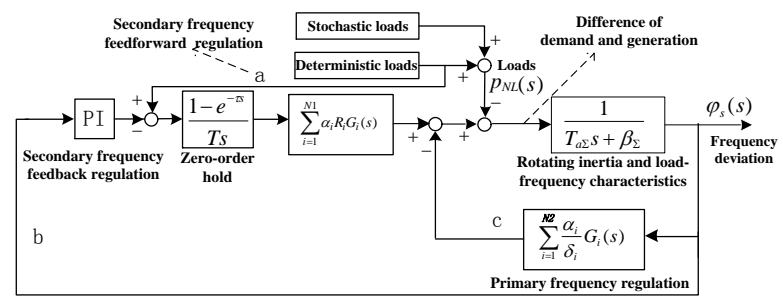

Fig.2. The control block diagram of single-region power grid 


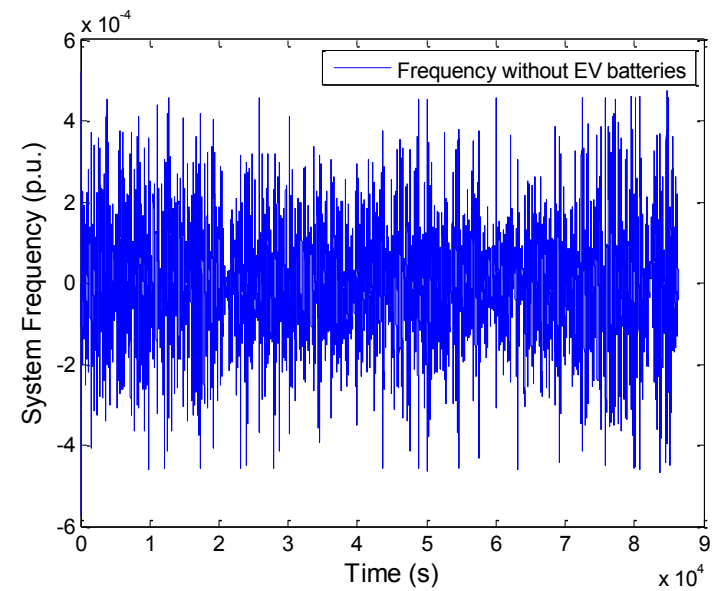

Fig.3. Dynamic frequency curve of traditional power grid

On account of quick response, in this model, EVs batteries are one part of primary frequency system by using negative feedback link. The other parts of the system are the same as the original one. But the parameters of Integral element and differentiation element are modified to maintain system stability.The result of simulation shows that the maximum frequency deviation is around 0.002 based on per unit system frequency(figure 4). Compared with the system without EVs,the frequency regulation ability of the system has improved.

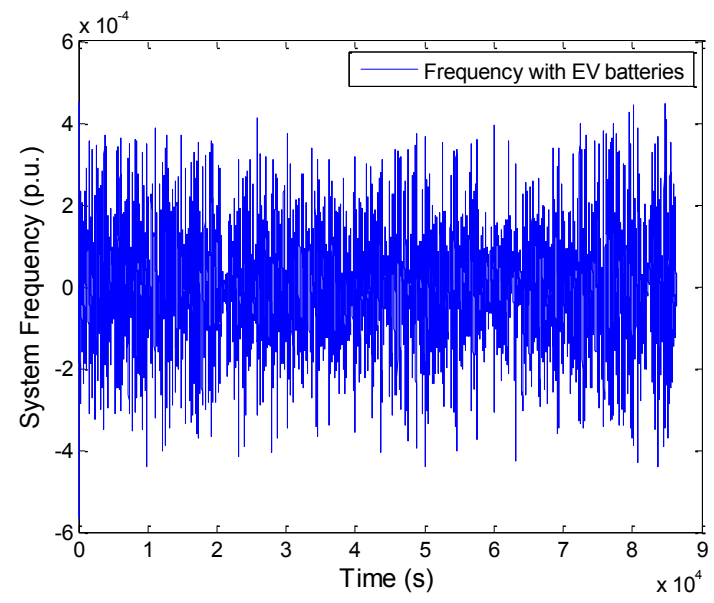

Fig.4. Dynamic frequency curve of power grid with EVs

Depending on charging/discharging situations, we adopt to two strageties, charging/discharging integration and charging/discharging separation.

Primary frequency control aims at random component of variable. In the long run, the load will change around zero. The EV batteries can keep enough power for longer. Under such background, in Stragety 1, EVs batteries can be seen as bi-directed transfer. Though this manner doesn't influence the battery energy a lot, it may shorten the lifespan because of frequent energy conversion. Also, once one generator of the power system is broken, the corresponding loads will be undertaken by Evs in some degree.This may cause lower energy of batteries, which may influence EV owners demand.The curves, which show power and energy of batteries, are as figure 5 and figure 6.

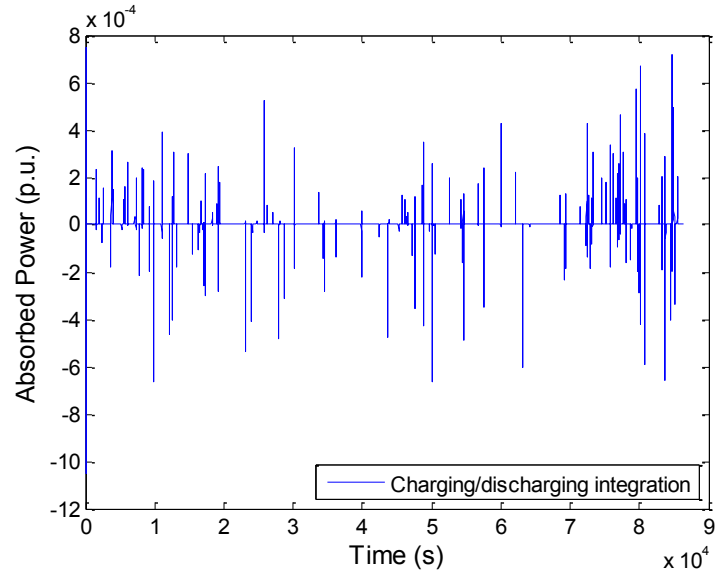

Fig.5. Dynamic power curve of EVs batteries in Strategy 1

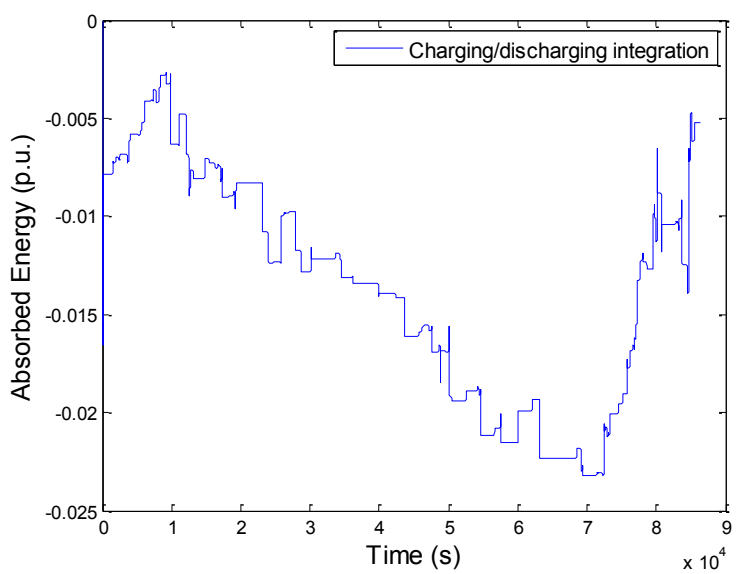

Fig.6. Dynamic energy curve of EVs batteries in Strategy 1

Frequent power conversion may influce the lifespan of EVs batteries by breaking structure of electrode. So,in Strategy 2, charging and discharging are separated into two groups to reduce transform number. The curves of input power,output power,input energy and output energy are as figure $7-10$. From the program ,the number of zero passage in this strategy is 165 .However,in strategy 1 ,this number is 168 .From the theory of cycle life method,

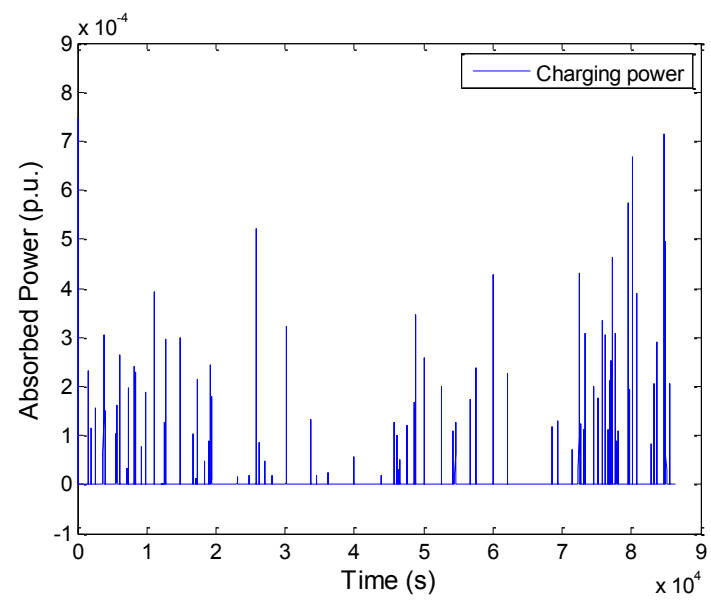

Fig.7. Dynamic input power curve of EVs batteries in Strategy 2 


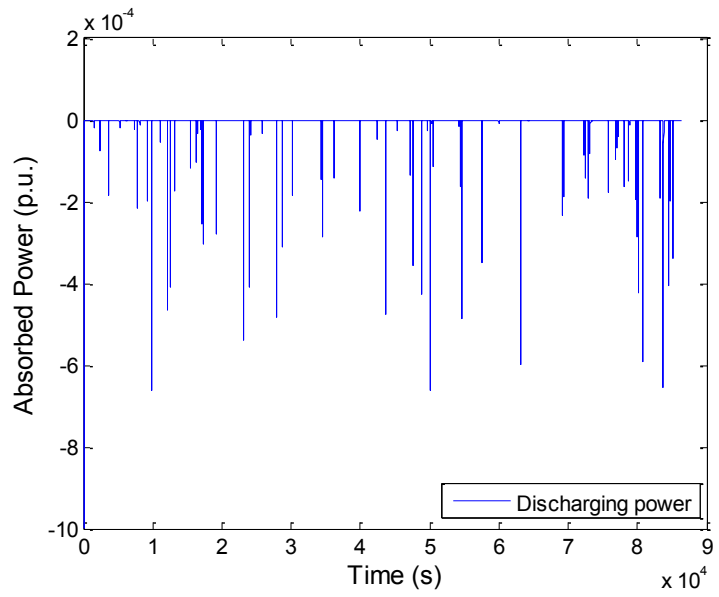

Fig.8. Dynamic output power curve of EVs batteries in Strategy 2

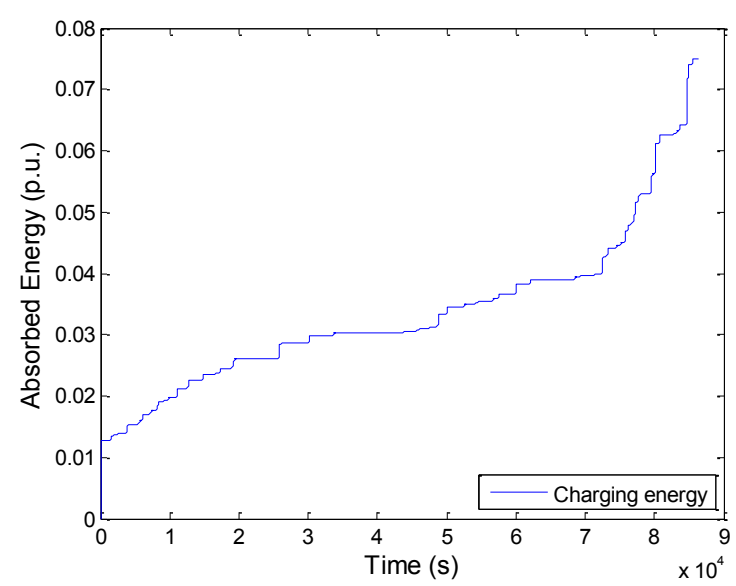

Fig.9. Dynamic input energy curve of EVs batteries in Strategy 2

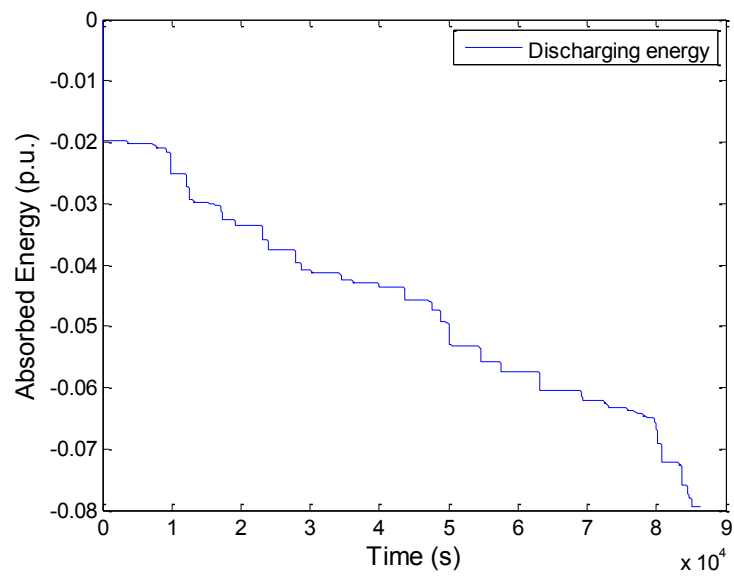

Fig.10. Dynamic output energy curve of EVs batteries in Strategy 2

After simulation, the matrix of per unit value of power can be obtained(figure 5,7,8). Then we can get the actual power and the current (the voltage is $3.6 \mathrm{~V}$ ). $B_{i}$ can be calculated. From figure 6, 9 and 10, we can get DOC and DOD of the batteries. According to the formula above, $A h_{\text {period_time_i }}$ can be obtained. Finally, we can get the capacity loss of the batteries.And the results are shown in Table I.
Table I.- Capacity loss of EVs batteries in one cycle $\left(25^{\circ} \mathrm{C}\right)$

\begin{tabular}{lc}
\hline \multicolumn{1}{c}{ Strategy } & Capacity loss \\
\hline $\begin{array}{l}\text { Strategy 1(Charging/discharging } \\
\text { integrated) }\end{array}$ & $0.0092 \%$ \\
$\begin{array}{l}\text { Strategy 2(Charging/discharging } \\
\text { separated:power in) }\end{array}$ & $0.0153 \%$ \\
$\begin{array}{l}\text { Strategy 2(Charging/discharging } \\
\text { separated:power out) }\end{array}$ & $0.0183 \%$ \\
\hline
\end{tabular}

\section{Conclusion}

Frequency characteristic of power grid can be improved by EVs.However, to guarantee the lifespan of batteries, the response to frequency deviation should be limited. In addition, the level of power should be proper to protect the structure of batteries. EVs can perform frequency regulation well. But the capacity loss of batteries in one day is about $0.0092 \%$. Thus, the power grid should compensate EVs owners. Also, For EVs with unidirectional regulation, through they can provide regulation services, the capacity loss is bigger.

\section{References}

[1] Yilmaz, M.; Krein, P.T., "Review of the Impact of Vehicle-to-Grid Technologies on Distribution Systems and Utility Interfaces," Power Electronics, IEEE Transactions on, Dec. 2013, vol.28, no.12, pp.5673-5689

[2] Dan, A.; Farkas, C.; Prikler, L., "V2G effects on frequency regulation and under-frequency load shedding in a quasi-islanded grid,"PowerTech (POWERTECH), 2013 IEEE Grenoble, 16-20 June 2013, pp.1-6

[3] Wenz, M.; Worn, Heinz, "Event-based Production Rules for Data Aggregation in Wireless Sensor Networks,"Multisensor Fusion and Integration for Intelligent Systems, 2006 IEEE International Conference on, Sept. 2006, pp.59-64

[4] John Wang, Ping Liu, Jocelyn Hicks-Garner, "Cyclelife model for graphite-LiFePO4 cells", Journal of Power Sources 196 (2011),pp.3942-3948

[5] Prabha Kundur. Power System Stability and Control. EPRI Power System Engineering Series, McGraw Hill(1994) 